\title{
MULTIOBJECTIVE ENGINEERING DESIGN OPTIMIZATION PROBLEMS: A SENSITIVITY ANALYSIS APPROACH
}

\author{
Oscar Brito Augusto ${ }^{1 *}$, Fouad Bennis ${ }^{2}$ and Stephane Caro ${ }^{3}$
}

Received August 5, 2011 / Accepted July 20, 2012

\begin{abstract}
This paper proposes two new approaches for the sensitivity analysis of multiobjective design optimization problems whose performance functions are highly susceptible to small variations in the design variables and/or design environment parameters. In both methods, the less sensitive design alternatives are preferred over others during the multiobjective optimization process. While taking the first approach, the designer chooses the design variable and/or parameter that causes uncertainties. The designer then associates a robustness index with each design alternative and adds each index as an objective function in the optimization problem. For the second approach, the designer must know, a priori, the interval of variation in the design variables or in the design environment parameters, because the designer will be accepting the interval of variation in the objective functions. The second method does not require any law of probability distribution of uncontrollable variations. Finally, the authors give two illustrative examples to highlight the contributions of the paper.
\end{abstract}

Keywords: multiobjective optimization, Pareto-optimal solutions, sensitivity analysis.

\section{INTRODUCTION}

Many engineering design problems are multiobjective by nature, because they often involve more than one design objective to be optimized. These design objectives impose potentially conflicting requirements on the technical and economic performance of a given system. A designer must formulate an optimization problem with multiple objectives if he/she wishes to study the tradeoffs that exist between these conflicting objectives and to explore their design options.

Multiobjective engineering design problems often have design parameters with uncontrollable variations due to noise or uncertainties. Such variations can affect outcomes significantly, such as the performances of objective functions and/or the feasibility of the Pareto optimal solutions.

\footnotetext{
*Corresponding author

${ }^{1}$ Escola Politécnica da Universidade de São Paulo - E-mails: oscar.augusto@poli.usp.br / obaugust@usp.br

2 École Centrale de Nantes, Institut de Recherche en Communications et Cybernétique de Nantes

- E-mail: fouad.bennis@ec-nantes.fr

${ }^{3}$ Institut de Recherche en Communications et Cybernétique de Nantes - E-mail: stephane.caro@irccyn.ec-nantes.fr
} 
A robust optimal solution is as good as possible with regard to the objective functions, and it offers the lowest possible sensitivity to variations in design variables and design parameters. In practice, all engineering designs are sensitive to uncertainties that can arise from manufacturing operations, variations in material properties, the operating environment and other reasons. Moreover, non-robust designs can be expensive to produce or to operate and can fail frequently in service.

Figure 1 illustrates the solution of a single-objective robust optimization problem. The performance function $f(x)$ is minimum when the design variable $x$ is equal to $x_{\text {opt }}$. However, the sensitivity of $f(x)$ to variations in $x_{o p t}$ is significant. Indeed, $\Delta f_{\text {opt }}$, which depicts the range of variations in $f(x)$ for a given range of variations in $x$ around $x_{o p t}$, is large. On the contrary, $x_{r o b}$ is a local minimum of function $f(x)$, and the sensitivity of $f(x)$ to variations in $x_{r o b}$ is very small. Indeed, $\Delta f_{r o b}$, which depicts the range of variations in $f(x)$ for a given range of variations in $x$ around $x_{r o b}$, is small. In fact, $\Delta f_{r o b}<\Delta f_{o p t}$. Accordingly, $x_{r o b}$ is a good solution to the single-objective robust optimization problem. In the case of a multiobjective optimization problem, a robust optimum solution may be located in the neighborhood of the Pareto front. Such a solution should have as little sensitivity as possible to uncertainties, because it cannot violate any constraint and/or acceptable known variations in design objectives in the presence of uncertainties. In this context, the purpose of this paper is to define a methodology to help the designer choose one or several robust optimum solution(s) when he/she must address a multiobjective robust design optimization problem.

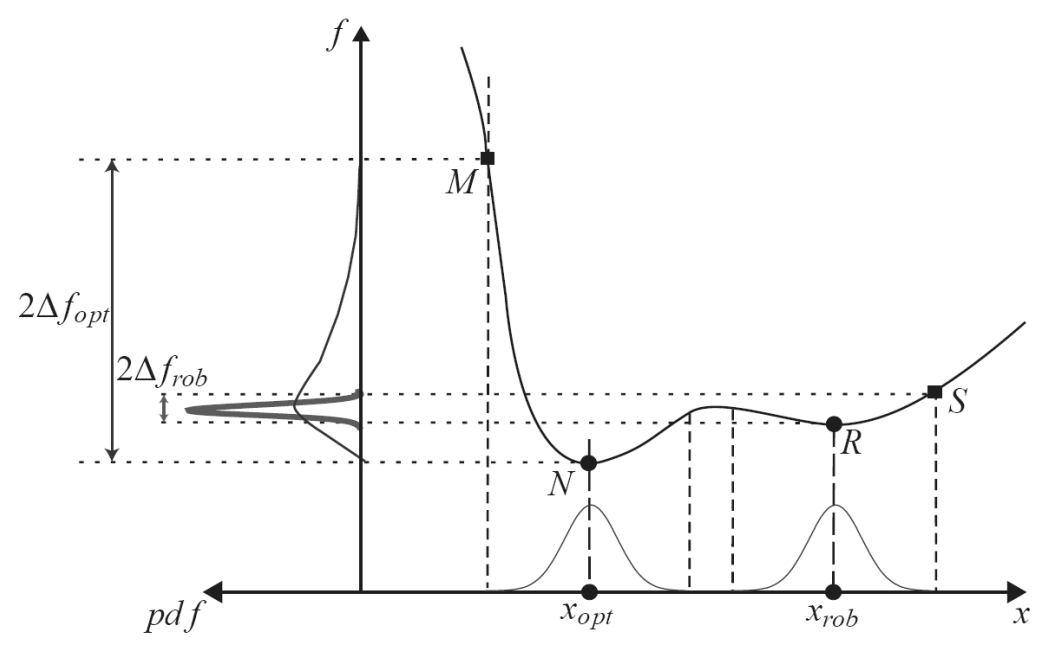

Figure 1 - A robust solution vs. an optimal solution.

First, the authors provide the formulations of a multiobjective optimization problem, a robust design problem and a multiobjective robust optimization problem. Then, a robustness index based on the sensitivity first order Jacobian of the objective functions is introduced, and the Pareto robustness concept is defined to deal with a multiobjective robust optimization problem. 
Then, two illustrative examples highlight the paper's contributions. Finally, the conclusions are discussed.

\section{Nomenclature}

$f_{i}(\mathbf{X}) \quad i$ th objective function

$\mathbf{f}(\mathbf{X}) \quad$ vector of objective functions

$g_{j}(\mathbf{X}) \quad j$ th inequality constraint function

KKT Karush-Khun-Tucker

$\mathbf{J}_{\mathrm{S}} \quad$ global sensitivity Jacobian matrix

$\mathbf{J}_{\mathrm{X}} \quad$ sensitivity Jacobian matrix related to the design variables

$\mathbf{J}_{\mathrm{p}} \quad$ sensitivity Jacobian matrix related to the design parameters

$k \quad$ number of objective functions

$m \quad$ number of inequality constraint functions

$n \quad$ number of decision variables

$q \quad$ number of design parameters

$p_{i} \quad i$ th design parameter

p vector of design parameters

$\mathbf{p}_{\text {inf }}, \mathbf{p}_{\text {sup }} \quad$ lower and upper bounds of the design parameters

$R(\mathbf{v}) \quad$ robustness index associated with the design variables $\mathbf{X}$ and the parameters $\mathbf{p}$

$\mathbb{S} \quad$ feasible region in the decision space

$S \quad$ sensitivity of the objective function

S diagonal matrix with the singular values in the singular value decomposition

$\mathbf{U}$ unitary matrix, expressed in the function space, in the singular value decomposition

$\mathbf{v} \quad$ vector joining the design variables $\mathbf{X}$ and the parameters $\mathbf{p}$

$\mathbf{V}$ unitary matrix expressed in the decision space in the singular value decomposition

$x_{i} \quad i$ th decision variable

$\overline{x_{i}} \quad$ average of the ith decision variable included in the optimal set

$\mathbf{X}$ decision or design variables vector

$\mathbf{X}^{*} \quad$ non-dominated solution of a multiobjective optimization problem

$\mathbf{X}_{\text {inf }}, \mathbf{X}_{\text {sup }} \quad$ lower and upper bounds in the decision space

$\lambda_{j}$

$\Delta x_{i_{\mathbf{0}}}$

$\Delta p_{i_{\mathbf{0}}}$

$\Delta f_{i_{\mathbf{0}}}$

$\lambda$

weighting factor for the $j$ th inequality constraint gradient in the $K K T$ condition

$\sigma_{i}$ interval of a known uniformly distributed variation of the $i$ th design variable interval of a known uniformly distributed variation of the $i$ th design parameter acceptable variation in the $i$ th objective function due to $\Delta \mathbf{v}$ uncertainties vector of $\lambda_{j s}$ standard deviation for the $i$ th decision variable included in the optimal set

$\omega_{i}$ weighting factor for the $i$ th objective function gradient in the $K K T$ condition vector of $\omega_{i s}$

$\nabla \quad$ gradient operator 


\section{Definitions of problems}

In this section, the formulations of (i) a multiobjective optimization problem, (ii) a robust design problem and (iii) a multiobjective robust optimization problem are given.

\subsection{Multiobjective optimization problem}

A general multiobjective optimization problem attempts to find the design variables $\mathbf{X}$ that optimize a vector objective function $\mathbf{f}(\mathbf{X})$ over the feasible design space $\mathbb{S}$. The determination of a set of non-dominated solutions, the Pareto optimum solutions or non-inferior solutions $\mathbf{X}^{*}$ can achieve a compromise among several objective functions. The problem formulation is defined as follows:

$$
\begin{array}{cl}
\text { minimize: } & \mathbf{f}(\mathbf{X}) \\
\text { subject to: } & g_{i}(\mathbf{X}) \leq 0, \quad i=1,2, \ldots m . \\
& \mathbf{X}_{\inf } \leq \mathbf{X} \leq \mathbf{X}_{\text {sup }}
\end{array}
$$

where $\mathbf{f}(\mathbf{X})=\left[f_{1}, f_{2}, f_{3}, \ldots, f_{k}\right]^{T}: \mathbb{R}^{n} \rightarrow \mathbb{R}^{k}$, with $f_{i}(\mathbf{X}): \mathbb{R}^{n} \rightarrow \mathbb{R}$ as a vector with the values of objective functions to be minimized. $\mathbf{X}$ is the vector that contains the design variables, also called decision variables, defined in the space $\mathbb{R}^{n} . \mathbf{X}_{\text {inf }}$ and $\mathbf{X}_{\text {sup }}$ are respectively the lower and upper bounds of the design variables. $g_{i}(\mathbf{X}): \mathbb{R}^{n} \rightarrow \mathbb{R}$ represents the $i^{\text {th }}$ inequality constraint function. Equations (1b) and (1c) define the region of feasible solutions, $\mathbb{S}$, in the decision variable space. The constraints $g_{i}(\mathbf{X})$ are "less than or equal" functions in view of the fact that "greater or equal" functions may be converted to the first type if they are multiplied by minus 1. Similarly, the problem deals with the "minimization" of functions $f_{i}(\mathbf{X})$, given that function "maximization" can be transformed into the former by multiplying it by minus 1 .

\subsubsection{Pareto optimal solution}

The notion of optimum in the context of solving multiobjective optimization problems is known as "Pareto optimal". A solution is said to be Pareto optimal if there is no alternative to improving one objective without worsening at least one other, that is, the feasible point $\mathbf{X}^{*} \mathbb{S}$ is Pareto optimal when there is no other feasible point $\mathbf{X} \in \mathbb{S}$ so $\forall i, j, f_{i}(\mathbf{X}) \leq f_{i}\left(\mathbf{X}^{*}\right)$ with strict inequality in at least one condition, $f_{j}(\mathbf{X})<f_{j}\left(\mathbf{X}^{*}\right)$.

Due to the conflicting nature of the objective functions, the Pareto optimal solutions are usually scattered in the region $\mathbb{S}$, a consequence of the solutions being unable to minimize the objective functions simultaneously. Solving the optimization problem achieves a set of Pareto optimal solutions defined in the decision space, after which an image of the objective functions, along with the Pareto front, is calculated over the set of optimal solutions.

In general, solving a multiobjective optimization problem is not as simple as solving any scalar problem. According to Schaffer (1985), Goldberg (1989) and Deb (2001), evolutionary algorithms are usually best suited to determining the Pareto front. 


\subsubsection{Necessary conditions for Pareto optimality}

Optimizing the multiobjective problems that are expressed by Eqs. (1a-1c) are of general character, because the equations represent the problem of single-objective optimization when $k=1$. According to Miettinen (1998), as in single-objective optimization problems, the solution $\mathbf{X}^{*} \in \mathbb{S}$ for the Pareto optimality must satisfy the Karush-Kuhn-Tucker condition, expressed as:

$$
\begin{aligned}
& \sum_{i=1}^{k} \omega_{i} \nabla f_{i}\left(\mathbf{X}^{*}\right)+\sum_{j=1}^{m} \lambda_{j} \nabla g_{j}\left(\mathbf{X}^{*}\right)=0 \\
& \lambda_{j} g_{j}\left(\mathbf{X}^{*}\right)=0 \\
& \lambda_{j} \geq 0 \\
& \omega_{i} \geq 0 ; \quad \sum_{i=1}^{k} \omega_{i}=1
\end{aligned}
$$

where $\omega_{i}$ is the weighting factor, positive, for the gradient of the $i$ th objective function, calculated at point $\mathbf{X}^{*}, \nabla f_{i}\left(\mathbf{X}^{*}\right) . \lambda_{j}$ represents the weighting factor for the gradient of the $j$ th inequality constraint function $\nabla g_{j}\left(\mathbf{X}^{*}\right)$. It is zero when the associated constraint function is not active, i.e., $g_{j}\left(\mathbf{X}^{*}\right)<0$.

It should be emphasized that the set of Eqs. (2a) to (2d) form the necessary conditions for $\mathbf{X}^{*}$ to be Pareto optimal.

\subsection{Robust design problem}

The concept of robust design was first used by Taguchi (1993). He introduced the concept of parameter design to improve the quality of a product whose manufacturing process involves significant variability or noise. Robust design aims at minimizing the sensitivity of performance to variations without controlling the causes of these variations. In the last decades, several authors contributed to the formulation and the improvement of robust design problems.

To deal with robustness, a set of design parameters $\mathbf{p}=\left[p_{1}, p_{2}, p_{3}, \ldots p_{q}\right]^{T}$ should be considered. Those parameters cannot be adjusted by the designer and are thus uncontrollable, such as the cost of the steel used in ship construction. The design variables

$$
\mathbf{X}=\left[x_{1}, x_{2}, x_{3}, \ldots x_{n}\right]^{T}
$$

can also be subjected to uncontrollable variations for the reasons of manufacturing errors, wearing or other uncertainties, although their nominal value is fixed.

A general multiobjective robust design optimization problem aims to find the design variables that optimize a vector objective function, $\mathbf{f}(\mathbf{X}, \mathbf{p})$, and to minimize its range of variations $\Delta \mathbf{f}(\mathbf{X}, \mathbf{p})=\left[\Delta f_{1}, \Delta f_{2}, \Delta f_{3}, \ldots \Delta f_{k}\right]^{T}$ over the feasible design space $\mathbb{S}$. The determination of a set of non-dominated solutions achieves a compromise among several objective functions that 
consider variations in the design variables and parameters. Calling $\mathbf{v}^{T}=\left[\mathbf{X}^{T} \mathbf{p}^{T}\right]$, the problem formulation can be defined as follows:

$$
\begin{aligned}
\text { minimize: } & \mathbf{f}(\mathbf{X}, \mathbf{p}), \\
& \boldsymbol{\Delta} \mathbf{f}(\mathbf{X}, \mathbf{p}) \\
\text { over } & \mathbf{X}=\left[x_{1}, x_{2}, x_{3}, \ldots x_{n}\right]^{T} \\
\text { subject to: } & g_{i}(\mathbf{X}, \mathbf{p})+\Delta g_{i}(\mathbf{X}, \mathbf{p}) \leq 0, \quad i=1,2, \ldots m . \\
& \mathbf{X}_{\text {inf }} \leq \mathbf{X} \leq \mathbf{X}_{\text {sup }} \\
& \mathbf{v}-\boldsymbol{\Delta} \mathbf{v}_{\text {inf }} \leq \mathbf{v} \leq \mathbf{v}+\Delta \mathbf{v}_{\text {sup }}
\end{aligned}
$$

All sets of equation (3) are general for search robust solutions of multiobjective optimization problems.

Sundaresan et al. (1993) developed a procedure that incorporates uncertainties in design variables and variations in constraints due to these uncertainties. Chase et al. (1996) presented the direct linearization method for tolerance analyses of 2D and 3D mechanical assemblies. Chen et al. (1996) studied two broad categories of problems, namely, (i) Type 1 problems, which minimize variations in performance caused by variations in noise factors (uncontrollable parameters), and (ii) Type 2, which minimize variations in performance caused by variations in control factors (design variables). Ben-Tal and Nemirovski (1998, 2002) proposed a study of convex optimization problems for which the data, in the present notation $\mathbf{p}$, is not specified exactly. Instead, the data are known only to belong to a given uncertainty set. They developed models for uncertain Linear, Conic Quadratic and Semidefinite programming problems. Kalsi et al. (2001) introduced a technique to reduce the effects of uncertainty and incorporated flexibility in the design of complex engineering systems involving multiple decision makers. Parkinson (2000) used a deterministic method of robust design to determine the optimum nominal dimensions of an assembly in order to improve the assembly quality. Bertsimas et al. (2004) have proposed a robust constrained optimization method for linear programming problems where the matrix of coefficients belongs to a known uncertainty set that is bounded. They have shown that this kind of problem is still linear programming. Bertsimas and Sim (2004) also focused on linear programming problems, seeking to reduce the level of conservatism of the robust solutions in terms of probabilistic bounds of constraint violations. They have shown that their method retains the advantages of the linear framework and offers full control over the degree of conservatism for every constraint. Thus, their method provides a probabilistic guarantee that the robust solution will be feasible with high probability.

The solutions of the presently proposed methods will always be feasible, and all nominal values of problem parameters, $\mathbf{p}$, are known. For the first method, described in section 3.1, no additional information is needed to search the less sensitive solutions under variations of $\mathbf{p}$ and eventually variations in the decision variables $\mathbf{X}$. For the second method, described in the section 3.2, these variations must be bounded. Both of these methodologies can be incorporated in any nonlinear multiobjective optimization algorithm. 
In the next section, the authors propose a simplified approach to searching for less sensitive alternatives when solving multiobjective optimization problems.

\section{A SIMPLIFIED MULTIOBJECTIVE ROBUST OPTIMIZATION PROBLEM}

Given that a robust optimal solution is as good as possible with regard to the objective functions and that it is as least sensitive as possible to variations in design variables and design parameters, this section presents two methods where robust design alternatives are preferred over others during the multiobjective optimization process.

First, the designer chooses only the design variables and/or design parameters that are subject to variations. With this information, a Robustness Index is associated with each design alternative. This index is added as one more function to be optimized. In the second approach, the designer accepts variations in the performance functions, limited in fixed intervals, knowing a priori the range of variations in the design variables and in the design parameters.

Assuming that $\mathbf{f}$ is of class $C^{2}$ in $\mathbf{v}$, one can expand Eq. (3a) in the neighborhood of the point $\mathbf{v}_{0}$ and keep only the linear terms. Then, the following equation can be obtained:

$$
\begin{aligned}
& \delta \mathbf{f}=\mathbf{J}_{s} \delta \mathbf{v}+\mathbf{e}\left(\|\delta \mathbf{v}\|_{2}\right) \\
& \delta \mathbf{v}^{T}=\left[\delta \mathbf{X}^{T} \delta \mathbf{p}^{T}\right] \\
& \mathbf{J}_{x}=\partial \mathbf{f} / \partial \mathbf{X} \\
& \mathbf{J}_{p}=\partial \mathbf{f} / \partial \mathbf{p} \\
& \mathbf{J}_{s}=\left[\mathbf{J}_{x} \mathbf{J}_{p}\right]
\end{aligned}
$$

where $\|\cdot\|_{2}$ denotes the Euclidian norm operator and $\mathbf{e}(\mathbf{v})$ an error function. $\mathbf{J}_{s}$ is the global sensitivity Jacobian matrix, and it describes the effect of the variations in design variables and design parameters to the performance functions. $\delta \mathbf{X}$ and $\delta \mathbf{p}$ are the variations in the design variables and in the design parameters, respectively. $\mathbf{J}_{x}$ is the $(k \times n)$ sensitivity Jacobian matrix of $\mathbf{f}(\mathbf{v})$ with respect to $\mathbf{X}$, and $\mathbf{J}_{p}$ is the $(k \times q)$ sensitivity Jacobian matrix of $\mathbf{f}(\mathbf{v})$ with respect to $\mathbf{p}$, respectively. If variations in the design variables are not considered, then $\mathbf{J}_{s}=\mathbf{J}_{p}$. If variations in design parameters are not considered, then $\mathbf{J}_{s}=\mathbf{J}_{x}$.

Ignoring the error in Eq. (4a), an approach to a robust solution is defined as one that is as least sensitive as possible to any variations in the decision variables and design parameters in its neighborhood. Considering $\boldsymbol{\Delta} \mathbf{v}$ as a closed normalized unit hyper-sphere centered at point $\mathbf{v}$ in the Euclidean space $\mathbb{R}^{n+q}$, i.e., $\|\boldsymbol{\Delta} \mathbf{v}\|_{2}=1$, then $\mathbf{J}_{S}$ is a linear application that maps the hypersphere in a hyper-ellipsoid in the normalized function space, centered in $\mathbf{f}(\mathbf{v})$ and described by the variations $\Delta \mathbf{f}(\mathbf{v}) \in \mathbb{R}^{k}$. In Figure 2, three design alternatives are checked for their sensitivity in the decision variable space. Since the local perturbation in the neighborhood of point $A$ causes a large modification in the objective values, this alternative may not be as robust as the alternative $\mathrm{B}$, because the latter does not bring on a large change in objective values, even in the presence of a local perturbation in its vicinity. 
Suppose that point $\mathrm{C}$ in Figure 2 belongs to the Pareto set and that its image is on the Pareto front. If any constraint function is not active at this point, then the hyper-ellipsoid collapses one of its axes. Being a Pareto solution, point $\mathrm{C}$ satisfies Eq. (2a), and the objective function gradients are linearly dependent. Consequently, the variations in the performance functions occur only along the tangent to the Pareto front.

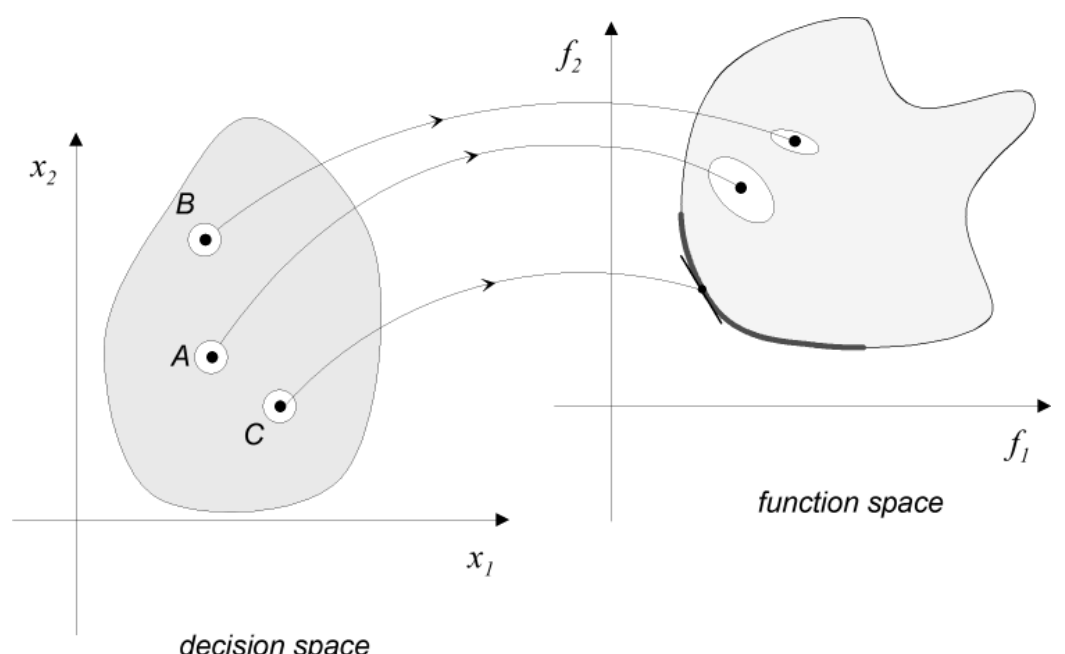

decision space

Figure 2 - The sensitivity Jacobian matrix transforms a unitary radius ball in the decision space into an ellipsoid in the function space.

\subsection{Robustness index}

The geometrical interpretation of Eq. (4a) can help one to define a Robustness Index in order to qualify a design alternative with regard to its robustness. The sensitivity Jacobian matrix can be decomposed by means of the singular value decomposition as follows:

$$
\mathbf{J}_{\mathbf{s}}=\mathbf{U S V}^{\mathbf{T}}
$$

where $\mathbf{U}$ is a $k$-by- $k$ orthogonal matrix, $\mathbf{S}$ is a $k$-by- $(k+q)$ diagonal matrix with non-negative real numbers and $\mathbf{V}^{\mathbf{T}}$ denotes the transposition of $\mathbf{V}$, which is a $(n+q)$-by- $(k+q)$ orthogonal matrix. The diagonal entries of $\mathbf{S}$ are known as the singular values of $\mathbf{J}_{\mathbf{s}}$. The singular value decomposition is a generalization of the decomposition of the eigenvalues and eigenvectors, and this decomposition is applied to a square matrix. If $\lambda_{i}$ is an eigenvalue of $\mathbf{J}_{\mathbf{s}} \mathbf{J}_{\mathbf{s}}^{\mathbf{T}}$, then the singular value $\sigma_{i}=\sqrt{\lambda_{i}}$. As a geometrical interpretation, the non-zero singular values, $\sigma_{i}$, are the lengths of the semi-axes of the hyper-ellipsoid represented in Figure 2, and the related vectors in $\mathbf{U}$ are the directions of these semi axes in the $\mathbb{R}^{k}$ space.

Let $S$ be the sensitivity of the objective functions to variations $\delta \mathbf{v} . S$ can be defined as the ratio of the Euclidean norm of variations in the objective functions, namely $\|\delta \mathbf{f}\|_{2}$, and the Euclidean norm of variations $\delta \mathbf{v}$, namely $\|\delta \mathbf{v}\|_{2}$. It turns out that $S$ is bounded by the smallest non-zero 
singular value $\sigma_{\min }$ and the largest singular value $\sigma_{\max }$ of its global sensitivity Jacobian matrix, $\mathbf{J}_{\mathbf{s}}$, namely,

$$
\sigma_{\min } \leq S=\frac{\|\delta \mathbf{f}\|_{2}}{\|\delta \mathbf{v}\|_{2}} \leq \sigma_{\max }
$$

Equation (6) shows that the lower $\sigma_{\max }$ is, the lower the upper bound of $S$ will be. Accordingly, the Euclidean norm of $\mathbf{J}_{\mathbf{s}}$, that is, its maximum singular value, can be used as a relevant Robustness Index:

$$
R(\mathbf{v})=\sigma_{\max }
$$

$R(\mathbf{v})$ makes sense if and only if the terms of $\mathbf{J}_{\mathbf{s}}$ are normalized, that is, if they have the same unit. Indeed, the singular values of $\mathbf{J}_{\mathbf{s}}$ cannot be compared if their units are different.

To illustrate the use of such a proposition, let us consider the following unconstrained minimization problem with two functions defined in $\mathbb{R}^{2}$ space:

$$
\begin{array}{ll}
\text { minimize: } & f_{1}\left(x_{1}, x_{2}\right)=c(-2,2)+c(2,2)+c(0,-2), \\
& f_{2}\left(x_{1}, x_{2}\right)=c(0,0) \\
\text { with } & c(a, b)=-\exp \left(-\left(\frac{x_{1}-a}{2}\right)^{2}-\left(\frac{x_{2}-b}{2}\right)^{2}\right)
\end{array}
$$

These two functions are illustrated in Figure 3.

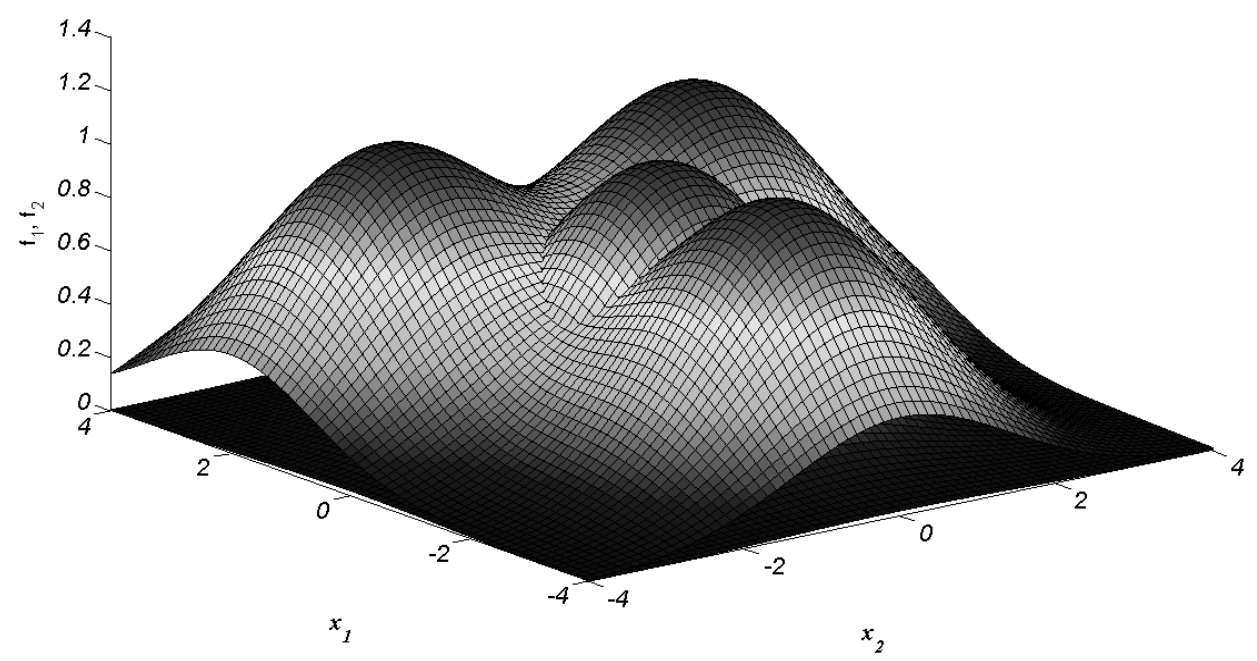

Figure 3 - Superimposed plot of exponential functions $-f_{1}\left(x_{1}, x_{2}\right)$ and $-f_{2}\left(x_{1}, x_{2}\right)$.

To find the non-dominated points, the Pareto dominance concept was applied to 5,000 randomly generated points over the interval $\left(x_{1}, x_{2}\right) \in[-4,4]$. The approximation of the Pareto set and the Pareto front are illustrated in Figures 4(a) and 4(b), respectively. In addition, Figure 4(a) shows the plots for the contours of both functions. The Pareto set is comprised of line segments that connect the minima of $f_{1}\left(x_{1}, x_{2}\right)$ to the minima of $f_{2}\left(x_{1}, x_{2}\right)$. 


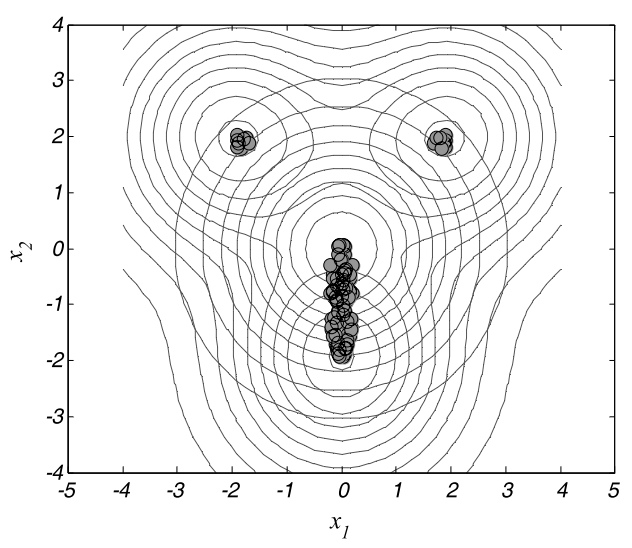

(a) Non-dominated points in decision space

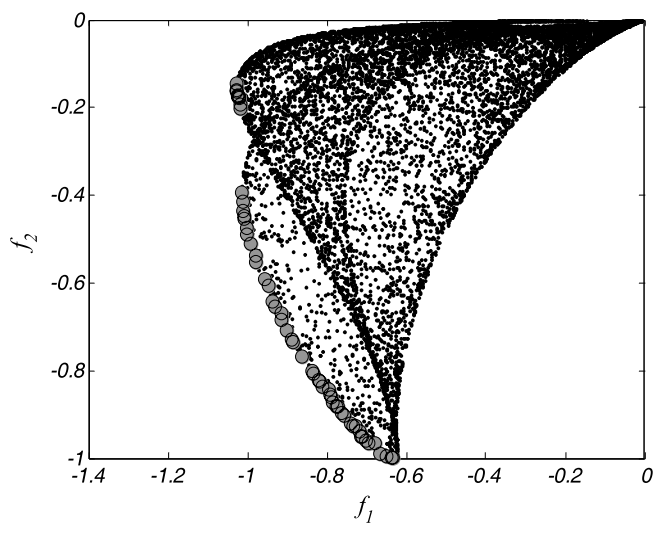

(b) Non-dominated points in function space

Figure 4 - Nominal approximations of the Pareto set and Pareto front for minimization of the exponential functions $f_{1}\left(x_{1}, x_{2}\right)$ and $f_{2}\left(x_{1}, x_{2}\right)$.

To search robust solutions, the authors applied the robust multi-objective optimization procedure by adding the robustness index, as defined by Eq. (7), as a third objective function. The robust problem can be written as

$$
\begin{array}{ll}
\operatorname{minimize}: & f_{1}\left(x_{1}, x_{2}\right)=c(-2,2)+c(2,2)+c(0,-2) \quad \text { and } \\
& f_{2}\left(x_{1}, x_{2}\right)=c(0,0) ; \quad \text { and } \\
& f_{3}\left(x_{1}, x_{2}\right)=R\left(x_{1}, x_{2}\right)=\sigma_{\max } \\
\text { with } \quad c(a, b)=-\exp \left(-\left(\frac{x_{1}-a}{2}\right)^{2}-\left(\frac{x_{2}-b}{2}\right)^{2}\right)
\end{array}
$$

The non-dominated points are shown in Figure 5. Clouds of points lie both near the nominal Pareto set and far away from it. In the specific problem, both functions are nearly flat in those regions. Accordingly, the robustness indexes for these points are very low, placing them as nondominated although their function values are non-optimal compared to those near the nominal Pareto set.

Given the flat regions in Figure 3, one can conclude that the robustness index, when included in the multi-objective optimization problem as an additional objective function to be minimized, permits the location of the less sensitive non-dominated alternatives. Moreover, it naturally disperses the nominal Pareto front of the original problem, causing the decision-making process to become even more difficult.

To overcome this difficulty, the authors suggest in the next section a complementary approach to dealing with robustness in multi-objective optimization problems. 


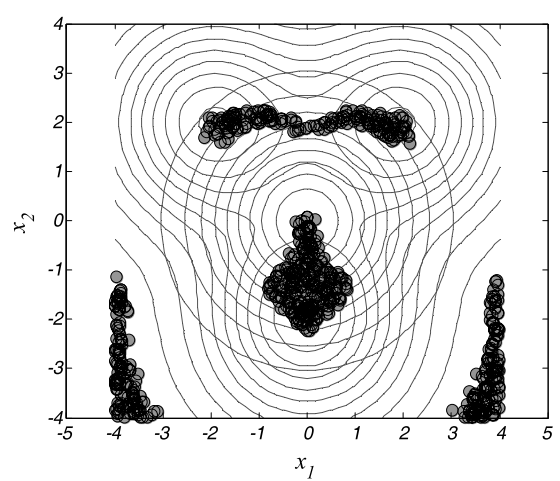

(a) Robust alternatives in the decision space

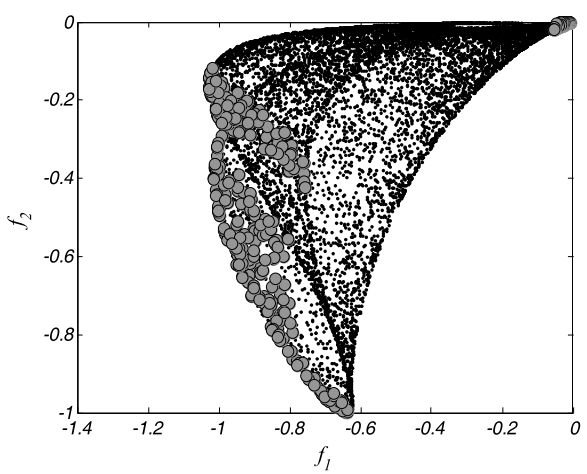

(c) $x-y$ view of the robust alternatives in the function space

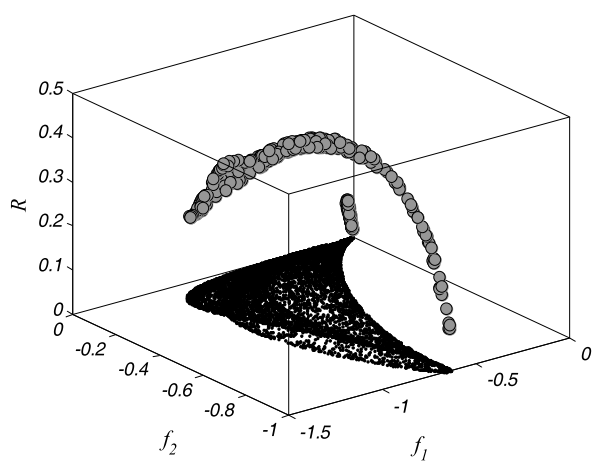

(b) Robust alternatives in the function space

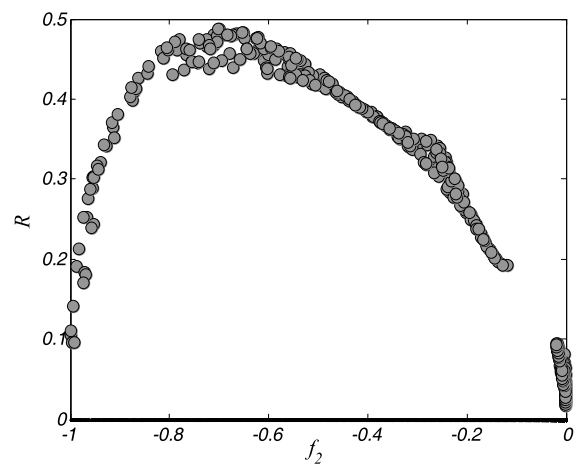

(d) $y-z$ view of the robust alternatives in the function space

Figure 5 - Non-dominated alternatives for the multi-objective robust optimization problem of the exponential functions $f_{1}\left(x_{1}, x_{2}\right)$, and $f_{2}\left(x_{1}, x_{2}\right)$ and the robustness index $R\left(x_{1}, x_{2}\right)$.

\subsection{Optimum with acceptable variations in the objective function}

To use this approach, the designer should know the bounds of variations in the design variables and in the design parameters, which are

$$
|\delta \mathbf{v}|=\Delta \mathbf{v}_{\mathbf{0}}
$$

The designer also accepts a tolerance for the variations in the objective functions, which are

$$
|\delta \mathbf{f}|_{a c c}=\Delta \mathbf{f}_{0}
$$

but he/she wants to keep all the constraints with consequent variations inside their initial bounds. For such conditions, the authors propose a robust multiobjective optimization problem as follows: 


$$
\begin{aligned}
\text { minimize: } & \mathbf{f}(\mathbf{v}) \\
\text { over } & \mathbf{X} \\
\text { subject to: } & g_{i}(\mathbf{v})+\Delta g_{i}(\mathbf{v}) \leq 0, \quad i=1,2, \ldots m . \\
& |\delta \mathbf{f}(\mathbf{v})|-\Delta \mathbf{f}_{\mathbf{0}} \leq 0 \\
& \mathbf{X}_{\text {inf }} \leq \mathbf{X} \leq \mathbf{X}_{\text {sup }} \\
\text { with } & |\delta \mathbf{v}|=\Delta \mathbf{v}_{\mathbf{0}}
\end{aligned}
$$

By using this approach, one can state the optimization problem with two exponential objective functions as:

$$
\begin{array}{cl}
\text { minimize: } & f_{1}\left(x_{1}, x_{2}\right)=c(-2,2)+c(2,2)+c(0,-2) \quad \text { and } \\
& f_{2}\left(x_{1}, x_{2}\right)=c(0,0) \\
\text { subject to: } \quad & \left|\delta\left(x_{1}, x_{2}\right)\right|-\Delta f_{10} \leq 0 \\
& \left|\delta f_{2}\left(x_{1}, x_{2}\right)\right|-\Delta f_{20} \leq 0 \\
& \Delta\left(x_{1}, x_{2}\right)_{0}=0.1 \\
\text { with } \quad & c(a, b)=-\exp \left(-\left(\frac{x_{1}-a}{2}\right)^{2}-\left(\frac{x_{2}-b}{2}\right)^{2}\right)
\end{array}
$$

where the acceptable function variations are set to 1 percent without loss of generality, and they include variations in the design variables that are equal to 10 percent of their nominal value. Figure 6 shows the Pareto optimal solutions plotted in the design space and the Pareto front approximation obtained by using the random walk over the design space.

Considering the acceptable values used, the robust Pareto front is less performing than the nominal one.

In the classical sensitivity analysis, the problems may have data (p, in the present notation) that are not specified exactly and are only known to belong to a given uncertainty set.

With the proposed methods, one can approach the engineering design optimization problem while considering the effects of uncertainty. The idea behind these methods is to consider that some data relating to engineering problems have variations around their nominal values. Moreover, the problems' data cannot be implemented exactly even if the data are certain and an optimal solution $\mathbf{X}^{*}$ can be computed exactly.

\section{APPLICATIONS}

This section presents two engineering examples to demonstrate the proposed multiobjective robust optimization. The first problem deals with the design of a vibrating platform. This problem includes six design variables with one being combinatorial; it also has five constraints and two uncontrollable parameters. This example should highlight the influence of the discrete variable in the robust search. The second problem deals with the conceptual design of a ship. 


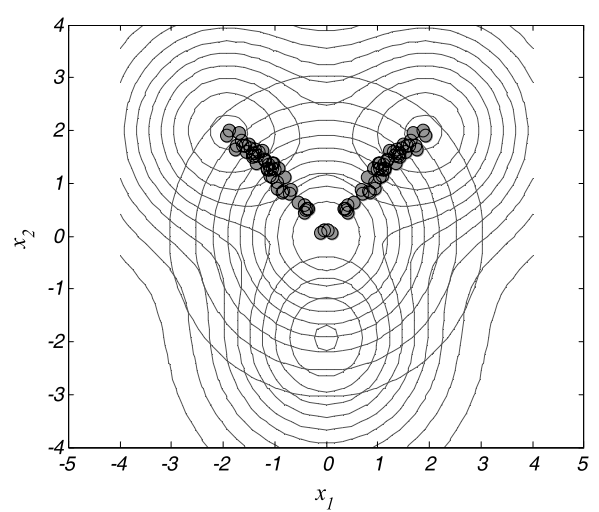

(a) Non-dominated alternatives in the decision space

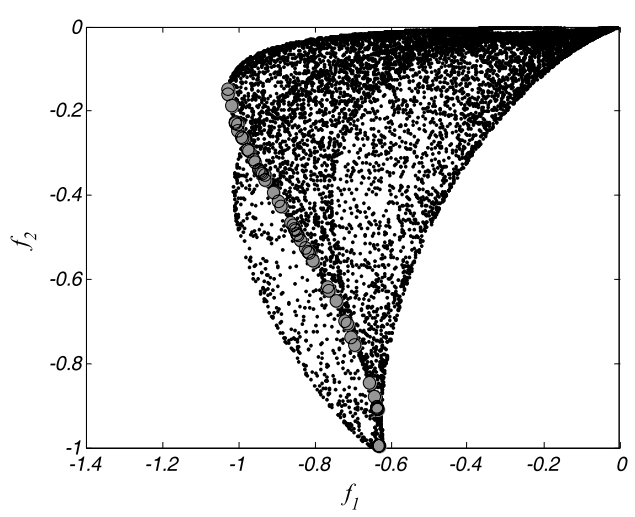

(b) Non-dominated points in the function space

Figure 6 - Non-dominated points for the robust multi-objective optimization problem of the exponential objective functions $f_{1}\left(x_{1}, x_{2}\right)$, and $f_{2}\left(x_{1}, x_{2}\right)$ with $\Delta\left(x_{1}, x_{2}\right)_{0}=0.1$ and $\Delta f_{10}=\Delta f_{20}=0.01$.

It contains six design variables, 21 constraints and three uncontrollable parameters, and it represents a more realistic problem that naval architects are likely to face.

To solve both problems, one will need a method to find the Pareto front for multiobjective optimization problems. The most widespread method in the literature is the genetic algorithm. Originally proposed by Holland (1975) for applications engaged with control theories, it was accepted quickly into numerous areas of engineering and science. Coello (2010) maintains an updated list of publications involving the genetic algorithm.

Many versions of genetic algorithms have served as meta-algorithms in the literature. The one that appears in this work was adapted from Deb et al. (2000), which is the Non-dominated Sorting Genetic Algorithm, version II (NSGA II). This version is easy to use and depends on only two parameters: the number of chromosomes in the population and the number of generations that this population will evolve. With each evolution, the non-dominated solutions in the population converge toward the Pareto optimal solutions.

\subsection{Problem 1: design of a vibrating platform}

To illustrate the proposed robust approach, the authors present the engineering problem adapted from Gunawan and Azarm (2005).

This problem aims to optimize the design of a platform modeled as a pinned-pinned sandwich beam with a vibrating motor on top, as shown in Figure 7. The platform has three layers (an inner layer, two middle layers sandwiching the inner layer and two outer layers sandwiching the inner and middle layers) of material. The layers must be comprised of three different materials that are named $A, B$ and $C$, and the choice of materials for the layers must be mutually exclusive so that two layers do not use the same material. However, the thickness of some layers can be null. 


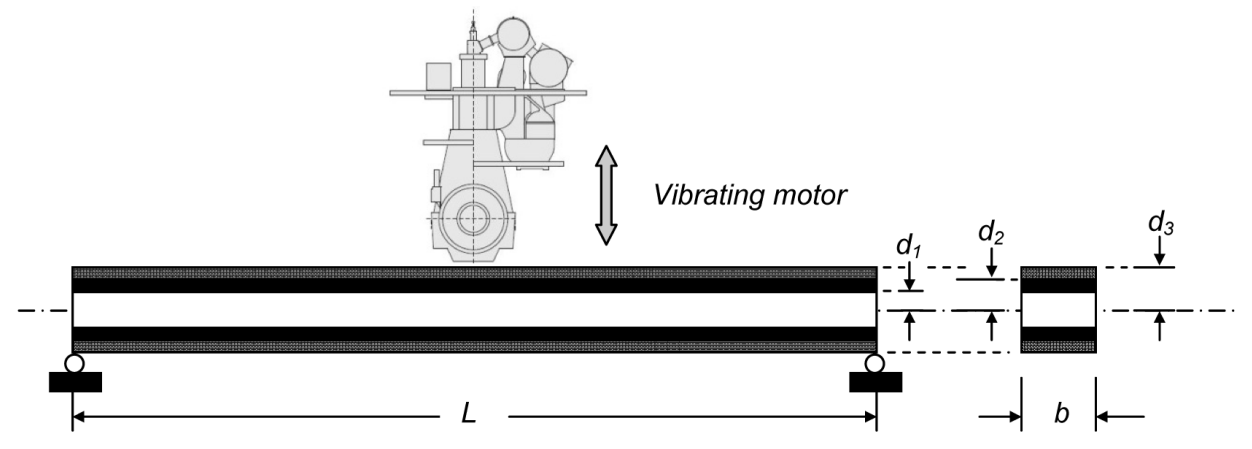

Figure 7 - Engine support platform.

The properties of the materials are shown in Table T1. In this table, $\rho$ is the mass density, $E$ is Young's modulus and $\mathrm{c}$ is the material cost per volume. The objectives are to minimize the total material cost used in such a platform and to maximize its natural frequency by controlling five sizing variables (continuous) and one combinatorial variable (discrete). The sizing variables are the width of the platform $(b)$, the length of the beam $(L)$ and the thicknesses of the three layers $\left(d_{1}, d_{2}\right.$ and $\left.d_{3}\right)$. The thicknesses of the middle and outer layers are represented as the difference between two sizing variables (e.g., the thickness of the middle layer is equal to $\left(d_{2}-d_{1}\right)$ ). The combinatorial variable is the choice of materials for the layers $(M)$. Since there are three possible material types, there are six possibilities for $M$ (starting from the inner layer outward): $\{A, B, C\},\{A, C, B\},\{B, A, C\},\{B, C, A\},\{C, A, B\}$ and $\{C, B, A\}$. The platform design is subjected to five constraints: the maximum weight of the platform and the lower and upper limits on the thickness of the middle and outer layers. The optimization formulation for this example is shown in Eq. (14). The notations $\left(\rho_{1}, \rho_{2}, \rho_{3}\right),\left(E_{1}, E_{2}, E_{3}\right)$ and $\left(c_{1}, c_{2}, c_{3}\right)$ refer to the density, Young's modulus and the material cost for the inner, middle and outer layers of the platform, respectively. The lower and upper bounds for the sizing variables are $0.05 \leq d_{1} \leq 0.5$, $0.2 \leq d_{2} \leq 0.5,0.2 \leq d_{3} \leq 0.6,0.35 \leq b \leq 0.5$ and $3 \leq L \leq 6$.

$$
\begin{aligned}
\text { maximize: } & f_{n}=\frac{\pi}{2 L^{2}} \sqrt{\frac{E I}{\mu}} \\
\text { minimize: } & \text { cost }=2 b L\left[c_{1} d_{1}+c_{2}\left(d_{2}-d_{1}\right)+c_{3}\left(d_{3}-d_{2}\right)\right] \\
\text { subject to: } & g_{1}=\mu L-2800 \leq 0 \\
& g_{2}=d_{1}-d_{2} \leq 0 \\
& g_{3}=d_{2}-d_{1}-0.15 \leq 0 \\
& g_{4}=d_{2}-d_{3} \leq 0 \\
& g_{5}=d_{3}-d_{2}-0.01 \leq 0 \\
\text { with } \quad & E I=\frac{2 b}{3}\left[E_{1} d_{1}^{3}+E_{2}\left(d_{2}^{3}-d_{1}^{3}\right)+E_{3}\left(d_{3}^{3}-d_{2}^{3}\right)\right] \\
& \mu=2 b\left[\rho_{1} d_{1}+\rho_{2}\left(d_{2}-d_{1}\right)+\rho_{3}\left(d_{3}-d_{2}\right)\right]
\end{aligned}
$$


Table T1 - Properties of the beam materials.

\begin{tabular}{|c|c|c|c|}
\hline & Material A & Material B & Material C \\
\hline$\rho\left(\mathrm{kg} / \mathrm{m}^{3}\right)$ & 100.0 & 2770 & 7780 \\
$E(G P a)$ & 1.6 & 70 & 200 \\
$c\left(\mathrm{~m}^{3}\right)$ & 500.0 & 1500 & 800 \\
\hline
\end{tabular}

It is assumed that there are uncontrollable variations in the density of material $\mathrm{A}\left(\rho_{A}\right)$ and cost of material $\mathrm{B}\left(c_{B}\right)$, and the optimum solutions must be as minimally sensitive as possible to these variations. Moreover, the designer wants to obtain the robust Pareto solutions to this problem for the nominal parameter values $\rho_{A}=100 \mathrm{~kg} / \mathrm{m}^{3}$ and $c_{B}=1500 \$ / \mathrm{m}^{3}$.

The variations in the parameters affect the two objective functions and the platform weight, and this effect is incorporated in the constraint function $g_{1}$. To take into account the feasibility of the robust search process, the following constraint functions were added.

$$
\begin{aligned}
& g_{6}=|\Delta \cos t|-\Delta \operatorname{cost}_{0} \leq 0 \\
& g_{7}=|\Delta f n|-\Delta f n_{0} \leq 0 \\
& g_{8}=g_{1}+\left|\Delta g_{1}\right| \leq 0
\end{aligned}
$$

where, for the sensitivity requirements, the acceptable relative variations in objective functions $\frac{\Delta f n_{0}}{f_{n}}$ and $\frac{\Delta \cos t_{0}}{\cos t}$ were arbitrarily set in the values shown in Figure 8 with maximum variation for the parameters of material $A$ defined by $\frac{\Delta \rho_{A}}{\rho_{A}}=\frac{\Delta c_{A}}{c_{A}}=0.05$. The variation related to the constraints expressed in Eqs. (15a-15c) were calculated for the extreme points of the interval composed by the parameter with its variation.

In Figures (8a-8b), the nominal Pareto set of the problem (without the uncontrollable variations) and the Pareto set obtained using the robust approach are displayed. When the robustness index is considered as the third objective function, the non-dominated points (square points) are dispersed over the function space, barely touching the nominal Pareto front. Therefore, the nominal Pareto front is not robust.

As expected, if the nominal solution is not robust, then different Pareto fronts will be obtained, because the acceptable variations in objective functions are modified. Table T2 shows the statistics for the results with different levels of these acceptable variations. This table displays some notable facts. First, in each Pareto set, all alternatives resulted with the same material sequence order. Second, the material order in the nominal Pareto set is from the cheapest to the most expensive material as well as from the inner, thicker layer to the external, thinner layer, respectively, which was also expected. Third, the platform cost variation due to maximum variations in material A is more relevant than the frequency variation, so one can say that the nominal Pareto front is robust from the point of view of natural frequency. Fourth, the nominal Pareto set has the platform cost variation at an average value of 4.51 percent with a standard deviation of 0.21 percent, which means that this set will be changed and the nominal Pareto front will be moved to a less performing region if the acceptable level of cost variations is set to the lower values, 
as shown in Figures (8c-8d). Finally, as long as the order of the material in the platform layers' cross section is acting as a design variable, the robust Pareto fronts will exhibit the behavior shown in Figure $8 \mathrm{c}$. The fronts with a relatively small variation in restrictive acceptable cost will fall in a better region than the fronts with more flexible bounds.

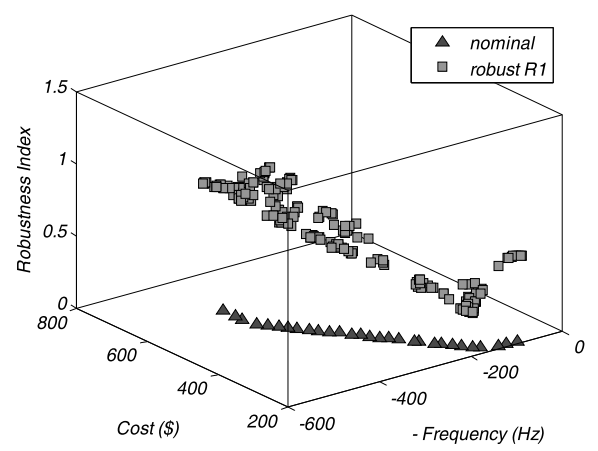

(a) Robust non-dominated points using robustness index

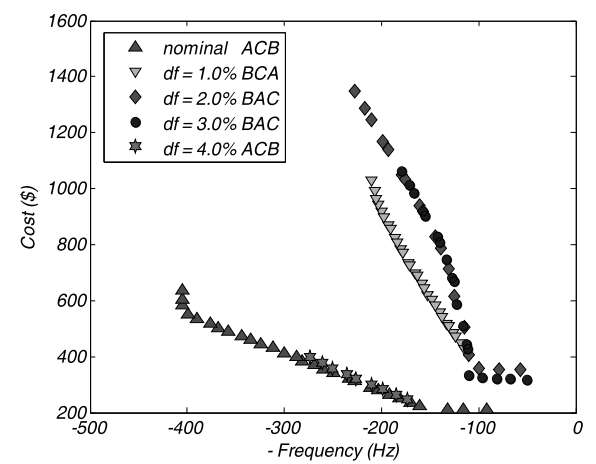

(c) Robust solutions with acceptable function variations and materials in the layers as design variables

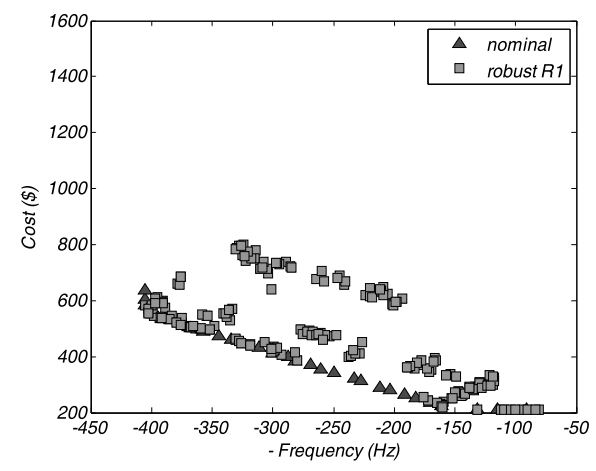

(b) xy view of the robust non-dominated points using robustness index

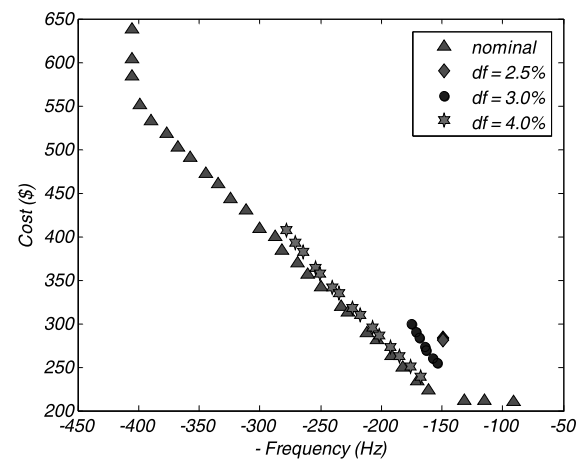

(d) Robust solutions with acceptable function variations and materials in the layers fixed as $\{A, C, B\}$

Figure 8 - Nominal and robust Pareto fronts of the platform design problem.

Table T2 - Statistics for design cases varying $\Delta f_{i_{o}} / f_{i}$ and material code as free design variable.

\begin{tabular}{|c|c|c|c|c|c|c|c|c|c|c|c|c|c|c|c|}
\hline$d v \rightarrow$ & \multicolumn{2}{|c|}{$b(m)$} & \multicolumn{2}{|c|}{$L(m)$} & \multicolumn{2}{|c|}{$d_{1}(m)$} & \multicolumn{2}{|c|}{$d_{2}(m)$} & \multicolumn{2}{|c|}{$d_{3}(m)$} & \multirow{2}{*}{$\begin{array}{l}\text { Material } \\
\text { code* }\end{array}$} & \multicolumn{2}{|c|}{$\Delta f_{n} / f_{n}(\%)$} & \multicolumn{2}{|c|}{$\Delta \cos t / \cos t(\%)$} \\
\hline$\Delta f_{i_{o}} \downarrow$ & $\bar{b}$ & $\sigma_{b}$ & $\bar{L}$ & $\sigma_{L}$ & $\overline{d_{1}}$ & $\sigma_{d_{1}}$ & $\overline{d_{2}}$ & $\sigma_{d_{2}}$ & $\overline{d_{3}}$ & $\sigma_{d_{3}}$ & & $\overline{\Delta f_{n}}$ & $\sigma_{\Delta f n}$ & $\overline{\Delta \cos t}$ & $\sigma_{\Delta \cos t}$ \\
\hline nominal & 0.35 & $2 \%$ & 3.00 & $0 \%$ & 0.33 & $29 \%$ & 0.35 & $30 \%$ & 0.35 & $31 \%$ & 2 & 0.55 & 0.48 & 4.51 & 0.21 \\
\hline $1 \%$ & 0.35 & $0 \%$ & 3.00 & $0 \%$ & 0.17 & $44 \%$ & 0.28 & $17 \%$ & 0.28 & $17^{\circ}$ & 4 & 0.00 & 0.00 & 0.00 & 0.00 \\
\hline $2 \%$ & 0.35 & $0 \%$ & 3.00 & $0 \%$ & 0.23 & $56 \%$ & 0.30 & $23 \%$ & 0.31 & $23^{\circ}$ & 3 & 0.05 & 0.06 & 0.8 & 0.82 \\
\hline $3 \%$ & 0.35 & $0 \%$ & 3.00 & $0 \%$ & 0.18 & $59 \%$ & 0.26 & $17 \%$ & 0.27 & $17 \%$ & 3 & 0.08 & 0.08 & 1.09 & 0.99 \\
\hline $4 \%$ & 0.35 & $0 \%$ & 3.00 & $0 \%$ & 0.25 & $17 \%$ & 0.28 & $16 \%$ & 0.28 & $17 \%$ & 2 & 0.20 & 0.02 & 3.99 & 0.01 \\
\hline
\end{tabular}

*code: $1=\{A, B, C\}, 2=\{A, C, B\}, 3=\{B, A, C\}, 4=\{B, C, A\}, 5=\{C, A, B\}$ and $6=\{C, B, A\}$. 
Furthermore, the proposed sensitivity approach is useful for characterizing robust Pareto fronts. With this approach, one can easily achieve the robust Pareto front. This approach does not require stochastic treatment for obtaining the variations, and it does not need a probability distribution for the variations in the design variables and design parameters.

\subsection{Problem 2: preliminary design of a bulk carrier}

The second application of the developed methodology is the preliminary design of a bulk carrier. The design of a vessel is not a trivial task. For decades, this problem has been handled in two ways. Some designers have adjusted a known design so that it meets new requirements, and others have relied on simplified mathematical models controlled by an optimization algorithm, which allow them to obtain the optimal solution based on previously established technical or economic criteria.

This work considers the second alternative with the aid of the mathematical model for designing bulk carriers, which was developed by Pratyush and Yang (1998) and presented in detail in Augusto et al. (2012) study. The model comprises a set of functions that define the vessel attributes. These functions constrain the design variables of the objective functions to be optimized as well as the space of these design variables. These functions characterize the technical and economic performance of the ship and allow designers to evaluate each design alternative. The economic performance of the ship refers to its annual unitary transportation cost and its annual transported cargo, and the technical performance of the ship refers to the functions of the vessel's design variables, including length, beam, depth, draft, block coefficient and speed, which are respectively ( $L, B, D, T, C b$ and $V_{K}$ ). Pratyush and Yang chose to minimize the annual transportation cost, maximize the amount of annual cargo and minimize the vessel's weight. The present work chose the optimization of the first two with no loss of generality. These two functions are conflicting, as shown in Figures 9 and 10.

The authors applied the proposed multiobjective robust optimization to the ship's design in order to consider the isolated variation in each design variable and in each design parameter for the two approaches. First, the Robustness Index was added as a third objective function to the original biobjective optimization problem. Then, the variations relative to the nominal value of the design variable and to the design parameter were arbitrarily preset at $\Delta x_{i_{0}}=1$ percent and 5 percent, respectively, and the consequent variations of the objective functions were limited to $\Delta f_{i_{\mathbf{0}}}$ and arbitrarily set at values ranging from 1 percent to 4 percent relative to their resultant or nominal values, depending on the case.

Figure 9 displays the results for the robust optimization related to the uncontrollable variations in each design variable. Each figure displays the nominal (non-robust) Pareto front, the robust Pareto front considering the Robustness Index as the third objective function to be minimized and the Pareto front considering different levels of acceptable variations in the objective functions as an effect of a design variable's uncontrollable variation around its nominal value. 


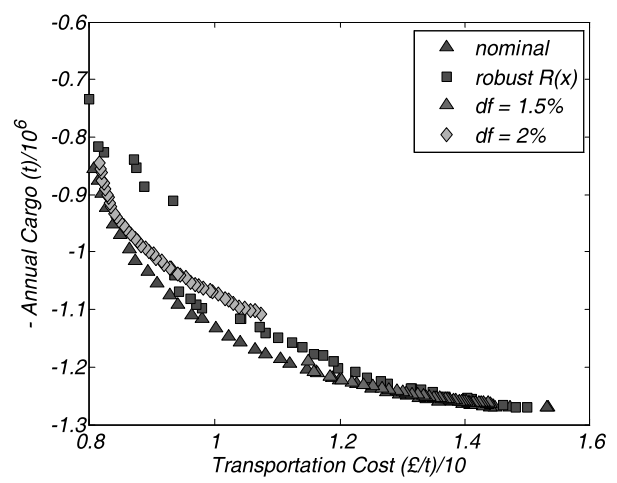

(a) Length

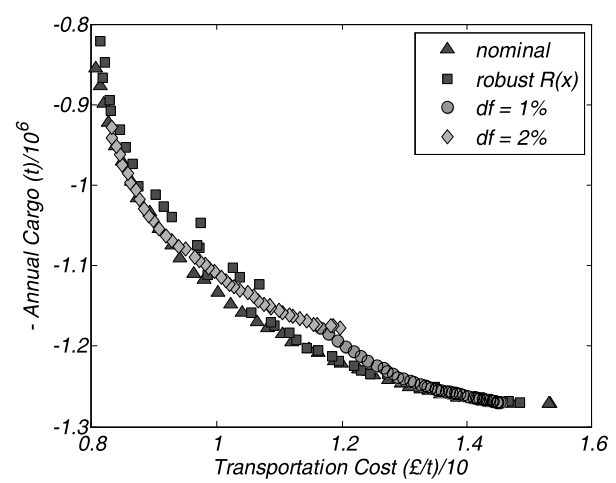

(c) Depth

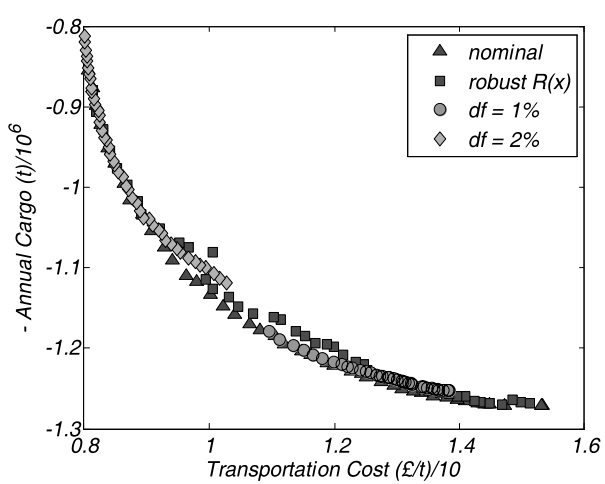

(e) Block coefficient

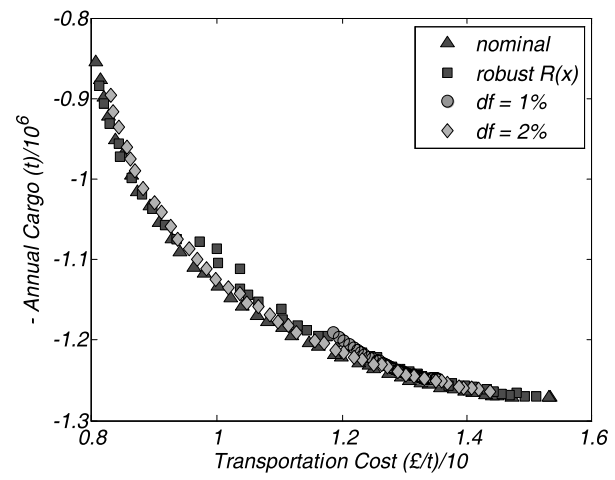

(b) Beam

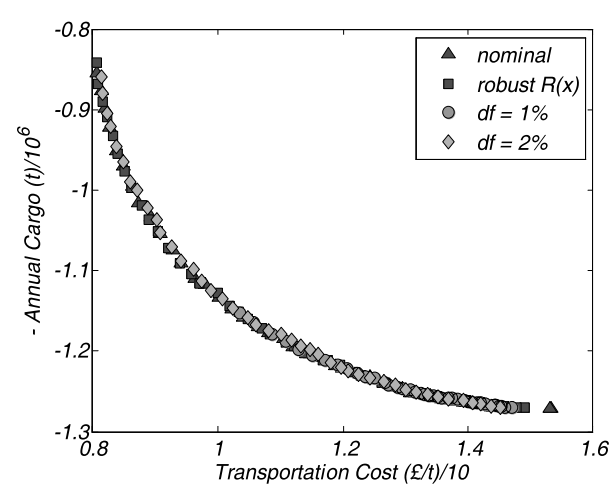

(d) Draft

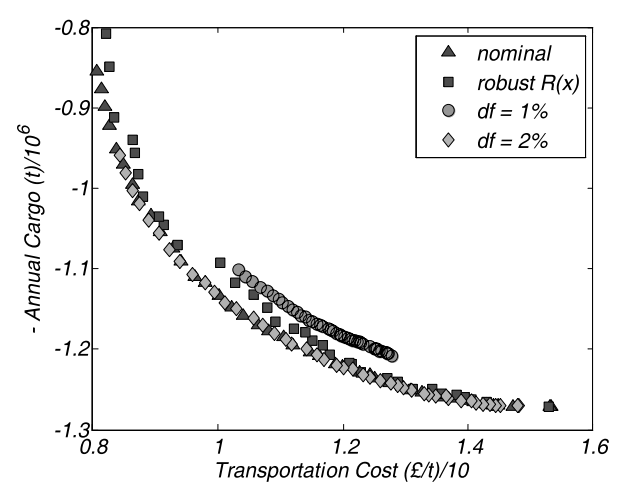

(f) Speed

Figure 9 - Nominal and robust Pareto fronts for the minimization of transportation $\operatorname{cost}\left(C_{T}\right)$ and maximization of annual transported cargo $\left(A_{C}\right)$, allowing isolated variations in the design variables of $(L, B, D$, $T, C b$ and $\left.V_{K}\right)$. 
Figure 10 shows the results for robust optimization related to variations in the arbitrarily chosen design parameters, namely round trip, fuel oil price and handling rate, since they can affect negatively the performance functions, depending on the uncertainties of their nominal values.

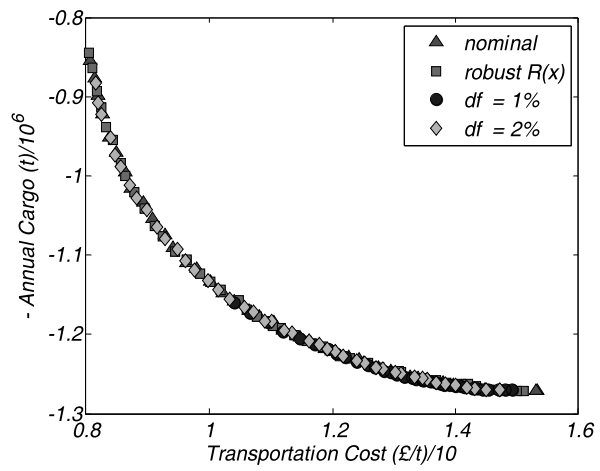

(a) Round trip

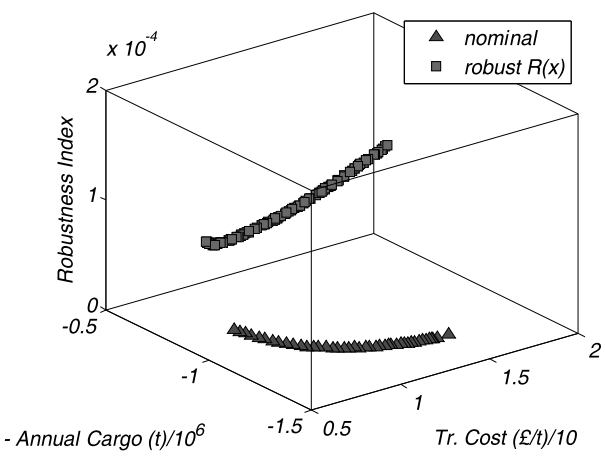

(c) Handling rate with robustness index

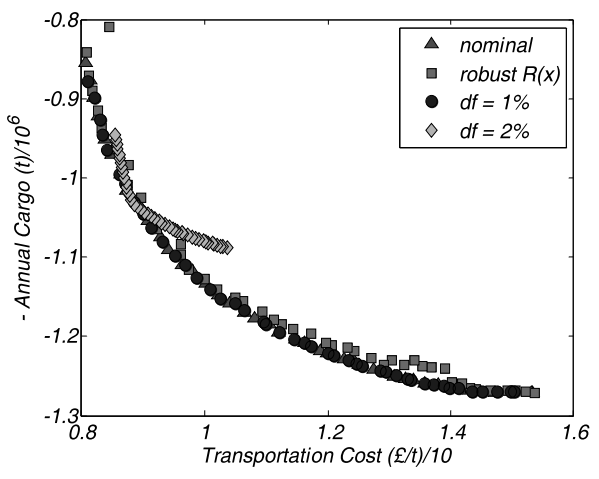

(b) Fuel oil price

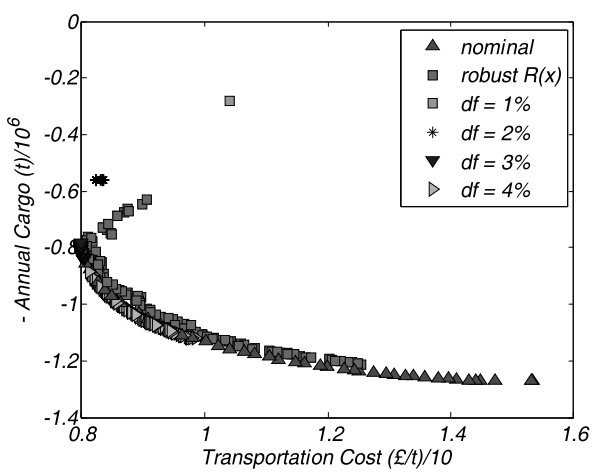

(d) Handling rate with acceptable function variations

Figure 10 - Nominal and robust Pareto fronts for the minimization of transportation cost $(C T)$ and maximization of annual transported cargo $(A C)$, allowing isolated variations in the design parameters of round trip, fuel oil price and handling rate.

Each figure shows the nominal (non-robust) Pareto front, the robust Pareto front considering the Robustness Index as the third objective function to be minimized and the Pareto front considering different levels of acceptable objective function variations due to an arbitrary parameter variation interval of $\Delta p_{i_{\mathbf{0}}}=5$ percent around the parameter's nominal value.

From both figures, it can be seen that variations in problem variables and problem parameters impact the extension and the performance of objective functions. More important is that the variation with the most impact in the nominal Pareto front is associated with the handling rate (HR) parameter, according to the results shown in Figure (10d). Due to this parameter, whose 
nominal value is set to $8,000 \mathrm{t} /$ day, the nominal Pareto front is very sensitive. For acceptable levels of variations in objective functions over the interval $\Delta f_{i_{0}} \in[1 \%, 3 \%]$, the respective Pareto fronts practically collapse to a single solution in each respective front. This single solution will be partially robust if the acceptable levels of variations are higher, namely $\Delta f_{i_{\mathbf{0}}}=4$ percent, when compared to those observed in the results obtained with uncontrollable variations in the design variables and other design parameters.

Therefore, this parameter plays an important role in the design process, because its impact on the objective functions can degrade drastically the performance of the designed ship.

\section{CONCLUSIONS}

Most engineering design problems are multiobjective and contain antagonistic objective functions. To solve such problems, many researchers developed methods that helped them to search for a general solution. They have frequently elected to use evolutionary methods to locate a set of solutions of multiobjective optimization problems. These algorithms provide a discrete picture of the Pareto front in the function space.

This paper introduced a new concept of a sensitivity index to perform multiobjective robust design optimizations, mainly when performance functions are highly sensitive to the variations in the design variables and in the design parameters.

To introduce the concept, the authors presented formulations of a multiobjective optimization problem, a robust design problem and a multiobjective robust optimization problem. A robustness index was introduced in order to classify the nominal Pareto front as either non-sensitive robust or not. This robustness index is based on the singular values of the sensitivity Jacobian matrix involving the objective functions, and it is considered an additional function to be minimized in the optimization problem. If the nominal Pareto front is not robust, then the new front, in view of the robustness index, will be scattered in the function space.

In addition, this paper proposed a supplementary method for searching for the robust Pareto front in instances where the design variables and design parameters have known uncontrollable variations bounded in single intervals and the designer will accept a range of these variations in the objective functions. During this search for optimal solutions, the designer constrains variations in objective functions to the acceptable intervals. The feasibility of the nominal problem is maintained once the effects of the variations in the constraint functions are considered.

Finally, two examples illustrated the contributions of the paper. First, the proposed method was applied to the design of an engine support platform, a problem with two objective functions, six design variables, five constraints and uncontrollable variations in the design parameters of material cost and material density. Then, a preliminary ship design, a problem with six design variables, two objective functions and twenty-one constraints, was conducted in a robust condition considering uncontrollable variations in the design variables and in the design parameters. The authors concluded that the nominal optimal set is not robust, because one of the ship's design parameters (the port handling rate) had a significant impact on the performance of the good 
under design. Given the results of both illustrations, the proposed methodology appears to be a simple and useful tool for conducting robust engineering designs.

\section{REFERENCES}

[1] Augusto O, Bennis F \& Caro S. 2012. A new method for decision making in multi-objective optimization problems. Pesquisa Operacional, in press.

[2] Ben-Tal A \& Nemirovski A. 1998. Robust convex optimization. Mathematics of Operations Research, 23(4): 769-805.

[3] Ben-Tal A \& Nemirovski A. 2002. Robust optimization - Methodology and applications. Mathematical Programming, 92(3): 453-480.

[4] Bertsimas D, Pachamanova D \& Sim M. 2004. Robust linear optimization under general norms. Operations Research Letters, 32: 510-516.

[5] Bertsimas D \& Sim M. 2004. The price of robustness. Operations Research, 52(1): 35-53.

[6] Caro S, Binaud N \& Wenger P. 2008. Sensitivity analysis of planar parallel manipulators. Proceedings of ASME Design Engineering Technical Conferences, August 3-6, New York, NY, U.S.

[7] Chase K, Gao J, Magleby SP \& Sorensen CD. 1996. Including geometric feature variations in tolerance analysis of mechanical assemblies. IIE Transactions, 28: 795-807.

[8] Chen W, Allen JK, Tsui K-L \& Mistree F. 1996. A procedure for robust design: minimizing variations caused by noise factors and control factors. ASME Journal of Mechanical Design, 118: $478-485$.

[9] Coello CAC. 2010. List of references on evolutionary multiobjective optimization. http://www.lania.mx/ ccoello/EMOO/EMOObib.html. Last accessed April 24, 2010.

[10] Deb K, Agrawal S, Pratab A \& Meyarivan T. 2000. A fast elitist non-dominated sorting genetic algorithm for multiobjective optimization. Technical Report 200001 NSGA-II, KanGAL.

[11] Deв K. 2001. Multiobjective Objective Optimization using Evolutionary Algorithms. San Francisco: Wiley \& Sons, Ltd.

[12] Giassi A, Bennis F \& Maisonneuve JJ. 2004. Multidisciplinary design optimization and robust design approaches applied to concurrent design. International Journal of Structural and Multidisciplinary Optimization, ISSMO, 28: 356-371.

[13] Goldberg D. 1989. Genetic Algorithms in Search and Machine Learning. Reading: Addison Wesley.

[14] Gunawan S \& AZARM S. 2005. Multiobjective robust optimization using a sensitivity region concept. Structural Multidisciplinary Optimization, 29: 50-60.

[15] Holland J. 1975. Adaptation in Natural and Artificial Systems. Ann Arbor: University of Michigan Press.

[16] KALSi M, HACKeR K \& Lewis K. 2001. A comprehensive robust design approach for decision trade-offs in complex systems design. ASME Journal of Mechanical Design, 121: 1-10.

[17] Miettinen KM. 1998. Nonlinear Multiobjective Optimization. New York: Springer. 
[18] PARKInSON DB. 2000. The application of a robust design method to tolerancing. ASME Journal of Mechanical Design, 22: 149-154.

[19] Pratyush S \& Yang JB. 1998. Multiple Criteria Decision Support in Engineering Design. New York: Springer.

[20] SCHAFFER JD. 1985. Multiple objective optimization with vector evaluated genetic algorithms. In: Proceedings of the First International Conference on Genetic Algorithms and their Applications [edited by J.J. Grefenstette], Psychology Press, 93-100.

[21] Sundaresan S, Ishit K \& Houser DR. 1993. A robust optimization procedure with variations on design variables and constraints. Advances in Design Automation, 1: 379-386.

[22] Taguchi G. 1993. On Robust Technology Development: Bringing Quality Engineering Upstream. New York: ASME Press. 